УДК 517.9

\author{
V. Kadets, M. Soloviova
}

\title{
A MODIFIED BISHOP-PHELPS-BOLLOBÁS THEOREM AND ITS SHARPNESS
}

V. Kadets, M. Soloviova. A modified Bishop-Phelps-Bollobás theorem and its sharpness, Mat. Stud. 44 (2015), 84-88.

Let $X$ be a real Banach space, $\varepsilon \in(0,1)$, and let $\left(x, x^{*}\right) \in S_{X} \times S_{X^{*}}$ with $x^{*}(x) \geqslant 1-\varepsilon$. Then, according to the modified Bishop-Phelps-Bollobás theorem, there exists $\left(y, y^{*}\right) \in S_{X} \times X^{*}$ such that $\left\|y^{*}\right\|=y^{*}(y)$, and $\max \left\{\|x-y\|,\left\|x^{*}-y^{*}\right\|\right\} \leqslant \sqrt{\varepsilon}$. We show that this theorem is sharp in a number of two-dimensional spaces, which makes a big difference with the original Bishop-Phelps-Bollobás theorem, where the only (up to isometry) two-dimensional space, in which the theorem is sharp, is $\ell_{\infty}^{(2)}$.

В. Кадец, М. Соловьёва. Модифицированная теорема Бишопа-Фелпса-Боллобаша и её точность // Мат. Студії. - 2015. - Т.44, №1. - С.84-88.

Пусть $X-$ банахово пространство, $\varepsilon \in(0,1)$ и $\left(x, x^{*}\right) \in S_{X} \times S_{X *}$ с $x^{*}(x) \geqslant 1-\varepsilon$. Тогда, согласно модифицированной теореме Бишопа-Фелпса-Боллобаша, существует такая пара $\left(y, y^{*}\right) \in S_{X} \times X^{*}$, что $\left\|y^{*}\right\|=y^{*}(y)$ и $\max \left\{\|x-y\|,\left\|x^{*}-y^{*}\right\|\right\} \leqslant \sqrt{\varepsilon}$. Мы доказываем, что эта теорема точна для целого семейства двумерных нормированных пространств, что существенно отличается от ситуации с исходной теоремой Бишопа-Фелпса-Боллобаша, где единственное (с точностью до изометрии) двумерное пространство, в котором теорема точна - это $\ell_{\infty}^{(2)}$.

1. Introduction. In this paper letter $X$ stands for a real Banach space. We denote, as usual $S_{X}$ and $B_{X}$ the unit sphere and the closed unit ball of $X$ respectively. A functional $x^{*} \in X^{*}$ attains its norm, if there is an $x \in S_{X}$ with $x^{*}(x)=\left\|x^{*}\right\|$. If $X$ is reflexive, then all $x^{*} \in X^{*}$ attain their norms, and in every non-reflexive space according to the famous James theorem (see [6, Chapter 1, theorem 3]) there are functionals that do not attain their norm. Nevertheless, even in a non-revlexive space there are "many" norm attaining functionals. Namely, the classical Bishop-Phelps theorem ( [1], see also [6, Chapter 1]) states that the set of norm attaining functionals on a Banach space is norm dense in the dual space, moreover, for every closed bounded convex set $C \subset X$ the collection of functionals that attain their maximum on $C$ is norm dense in $X^{*}$.

The fact that every functional can be approximated by norm attaining ones is quite useful, but sometimes one needs more. Namely, sometimes (in particular, when one works with numerical radius of operator) one needs to approximate a pair "element and functional" by a pair $\left(x, x^{*}\right)$ such that $x^{*}$ attains its norm in $x$. Such a modification of the BishopPhelps theorem was given by B. Bollobás ([2]). Nowadays this modification is called the Bishop-Phelps-Bollobás theorem.

2010 Mathematics Subject Classification: 46B20.

Keywords: Bishop-Phelps-Bollobás theorem; norm attaining functional.

doi:10.15330/ms.44.1.84-88

(C) V. Kadets, M. Soloviova, 2015 
Theorem 1 (Bishop-Phelps-Bollobás theorem [2]). Let $X$ be a Banach space. Suppose $x \in S_{X}$ and $x^{*} \in S_{X^{*}}$ satisfy $\left|1-x^{*}(x)\right| \leqslant \delta^{2} / 2(0<\delta<1 / 2)$. Then there exists $\left(y, y^{*}\right) \in$ $X \times X^{*}$ with $\|y\|=\left\|y^{*}\right\|=y^{*}(y)=1$, such that $\|x-y\|<\delta+\delta^{2}$ and $\left\|x^{*}-y^{*}\right\| \leqslant \delta$.

This statement can be a little bit improved (see [3] and [4]): instead of inequalities $\| x-$ $y \|<\delta+\delta^{2}$ and $\left\|x^{*}-y^{*}\right\| \leqslant \delta$ one can obtain a symmetric estimate $\max \left\{\|x-y\|,\left\|x^{*}-y^{*}\right\|\right\} \leqslant$ $\leqslant \delta$. Using the standard notation $\Pi_{\varepsilon}(X)=\left\{\left(x, x^{*}\right) \in X \times X^{*}:\|x\|=\left\|x^{*}\right\|=1, x^{*}(x) \geqslant\right.$ $1-\varepsilon\}, \varepsilon \geqslant 0$, and substituting $\varepsilon=\delta^{2} / 2$ one can re-write this improved statement as follows

Theorem 2 (Sharp Bishop-Phelps-Bollobás theorem). Let $X$ be a Banach space, $\varepsilon \in(0,2)$, and let $\left(x, x^{*}\right) \in \Pi_{\varepsilon}(X)$. Then there exists $\left(y, y^{*}\right) \in \Pi_{0}(X)$ such that

$$
\max \left\{\|x-y\|,\left\|x^{*}-y^{*}\right\|\right\} \leqslant \sqrt{2 \varepsilon} .
$$

The latter estimate is sharp for two-dimensional real space $\ell_{\infty}^{(2)}$, i.e. in $\mathbb{R}^{2}$ equipped with the norm $\left\|\left(x_{1}, x_{2}\right)\right\|=\max \left\{\left|x_{1}\right|,\left|x_{2}\right|\right\}$ (see [2] or [4, Example 2.5]). Moreover, for every $\varepsilon \in(0,1 / 2)$ Theorem 5.8 of [4] implies that $\ell_{\infty}^{(2)}$ is the only (up to isometry) twodimensional real space in which the estimate (1) is sharp. Even for spaces of higher dimensions $\ell_{\infty}^{(2)}$-subspaces play an important role in the Bishop-Phelps-Bollobás theorem sharpness: according to Theorem 5.9 of [4] if in a space $X$ estimate (1) cannot be improved, then $X$ contains almost isometric copies of $\ell_{\infty}^{(2)}$ (see [5, Theorem 3.3] for a simpler proof).

The key to the aforementioned sharp estimate in the Bishop-Phelps-Bollobás theorem is the following lemma which can be deduced from the Brøndsted-Rockafellar variational principle [8, Theorem 3.17] or from [7, Corollary 2.2] by R. R. Phelps.

Lemma 1. Let $X$ be a real Banach space, $\varepsilon>0$ and $\left(x, x^{*}\right) \in \Pi_{\varepsilon}(X)$. Then, for any $k \in(0,1)$ there exist $y^{*} \in X^{*}$ and $y \in S_{X}$ such that $\left\|y^{*}\right\|=y^{*}(y),\|x-y\| \leqslant \frac{\varepsilon}{k},\left\|x^{*}-y^{*}\right\| \leqslant k$.

From this lemma just substituting $k=\sqrt{\varepsilon}$ one can deduce the following modified version of the Bishop-Phelps-Bollobás theorem that appeared implicitly in the proof of $[8$, Theorem 3.18], pages 48-49:

Theorem 3 (Modified Bishop-Phelps-Bollobás theorem). Let $X$ be a Banach space, $\varepsilon \in(0,1)$, and let $\left(x, x^{*}\right) \in \Pi_{\varepsilon}(X)$. Then there exists $\left(y, y^{*}\right) \in S_{X} \times X^{*}$ such that $\left\|y^{*}\right\|=$ $y^{*}(y)$, and

$$
\max \left\{\|x-y\|,\left\|x^{*}-y^{*}\right\|\right\} \leqslant \sqrt{\varepsilon} .
$$

The improvement in the estimate (2) comparing to (1) appears because we do not demand $\left\|y^{*}\right\|=1$.

The aim of this short article is to investigate the sharpness of Theorem 3. We will demonstrate that this result is also sharp but surprisingly the presence of (almost) $\ell_{\infty}^{(2)}$-subspaces in $X$ is not a necessary condition for the sharpness of Theorem 3 in $X$. Namely, we present a collection of two-dimensional spaces with hexagonal unit sphere, in which (2) cannot be improved. We also discuss the sharpness of Lemma 1.

2. The example. Fix a $\rho \geqslant \frac{1}{2}$ and denote $X=X_{\rho}$ the linear space $\mathbb{R}^{2}$ equipped with the norm $\left\|\left(x_{1}, x_{2}\right)\right\|=\left\|\left(x_{1}, x_{2}\right)\right\|_{\rho}=\max \left\{\left|x_{1}-\frac{1-\rho}{\rho} x_{2}\right|,\left|x_{2}-\frac{1-\rho}{\rho} x_{1}\right|,\left|x_{1}+x_{2}\right|\right\}$.

In other words,

$$
\left\|\left(x_{1}, x_{2}\right)\right\|= \begin{cases}\left|x_{1}+x_{2}\right|, & \text { if } x_{1} x_{2} \geqslant 0 \\ \left|x_{1}-\frac{1-\rho}{\rho} x_{2}\right|, & \text { if } x_{1} x_{2}<0 \text { and }\left|x_{1}\right|>\left|x_{2}\right| \\ \left|x_{2}-\frac{1-\rho}{\rho} x_{1}\right|, & \text { if } x_{1} x_{2}<0 \text { and }\left|x_{1}\right| \leqslant\left|x_{2}\right| .\end{cases}
$$


and the unit ball $B_{\rho}$ of $X_{\rho}$ is the hexagon abcde $f$, where $a=(1,0) ; b=(0,1) ; c=(-\rho, \rho) ; d=$ $(-1,0) ; e=(0,-1)$; and $f=(\rho,-\rho)$.

The dual space to $X_{\rho}$ is $\mathbb{R}^{2}$ equipped with the polar to $B_{\rho}$ as its unit ball. So the unit ball $B_{\rho}^{*}$ of $X_{\rho}^{*}$ is the hexagon $a^{*} b^{*} c^{*} d^{*} e^{*} f^{*}$, where $a^{*}=(1,1) ; b^{*}=\left(-\frac{1-\rho}{\rho}, 1\right) ; c^{*}=\left(-1, \frac{1-\rho}{\rho}\right) ; d^{*}=$ $(-1,-1) ; e^{*}=\left(\frac{1-\rho}{\rho},-1\right)$; and $f^{*}=\left(1,-\frac{1-\rho}{\rho}\right)$. The norm on $X^{*}=X_{\rho}^{*}$ is given by the formula $\left\|\left(x_{1}, x_{2}\right)\right\|^{*}=\left\|\left(x_{1}, x_{2}\right)\right\|_{\rho}^{*}=\max \left\{\left|x_{1}\right|,\left|x_{2}\right|, \rho\left|x_{1}-x_{2}\right|\right\}$.

The corresponding spheres $S_{\rho}$ and $S_{\rho}^{*}$ are shown on Figures 1 and 2 respectively.

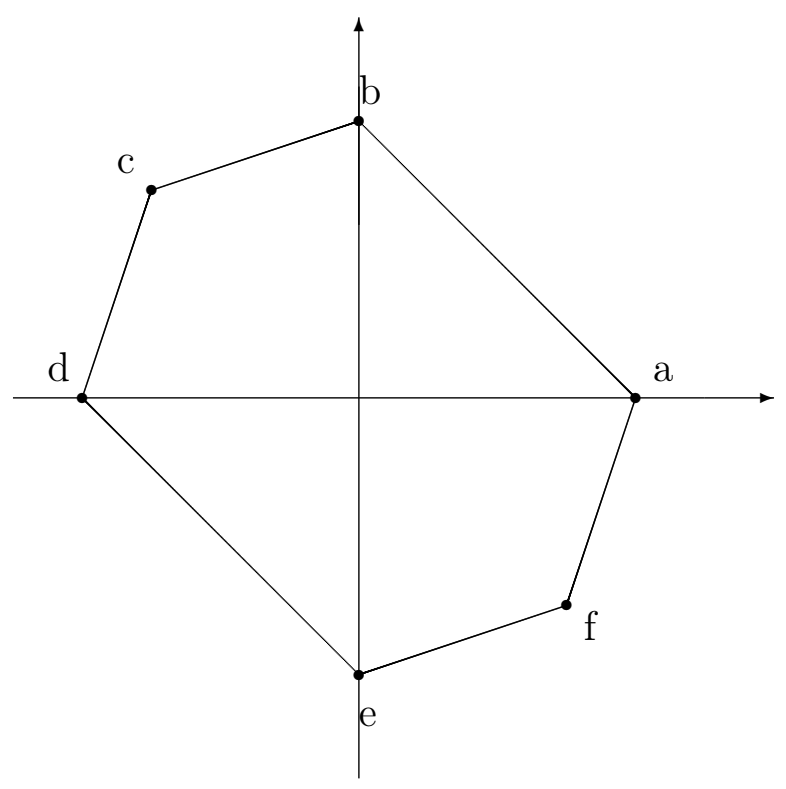

Figure 1

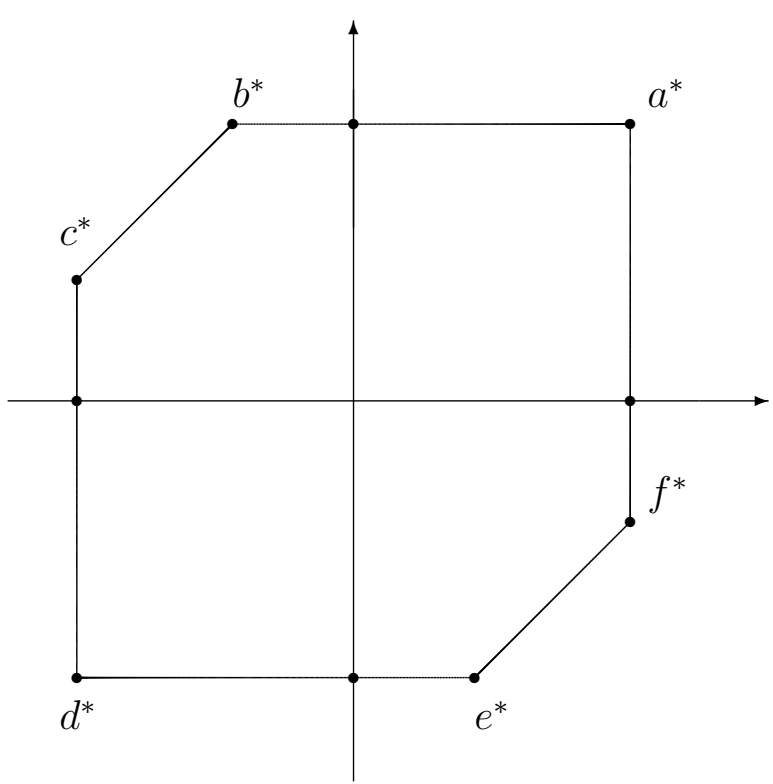

Figure 2

In the case of $\rho=\frac{1}{2}$ the sphere of $X_{\rho}$ reduces to the square abde, and consequently $X_{1 / 2}$ is isometric to the spaces $\ell_{1}^{(2)}$ and $\ell_{\infty}^{(2)}$. When $\rho>\frac{1}{2}$, the space $X_{\rho}$ is not isometric to $\ell_{\infty}^{(2)}$.

The set $\Pi_{0}\left(X_{\rho}\right)$ is the following polygon in $\mathbb{R}^{2} \times \mathbb{R}^{2}$ :

$$
\begin{gathered}
\Pi_{0}\left(X_{\rho}\right)=\left\{\left(a, x^{*}\right): x^{*} \in\left[f^{*}, a^{*}\right]\right\} \cup\left\{\left(x, a^{*}\right): x \in[a, b]\right\} \cup\left\{\left(b, x^{*}\right): x^{*} \in\left[a^{*}, b^{*}\right]\right\} \cup \\
\cup\left\{\left(x, b^{*}\right): x \in[b, c]\right\} \cup\left\{\left(c, x^{*}\right): x^{*} \in\left[b^{*}, c^{*}\right]\right\} \cup\left\{\left(x, c^{*}\right): x \in[c, d]\right\} \cup \\
\cup\left\{\left(d, x^{*}\right): x^{*} \in\left[c^{*}, d^{*}\right]\right\} \cup\left\{\left(x, d^{*}\right): x \in[d, e]\right\} \cup\left\{\left(e, x^{*}\right): x^{*} \in\left[d^{*}, e^{*}\right]\right\} \cup \\
\cup\left\{\left(x, e^{*}\right): x \in[e, f]\right\} \cup\left\{\left(f, x^{*}\right): x^{*} \in\left[e^{*}, f^{*}\right]\right\} \cup\left\{\left(x, f^{*}\right): x \in[f, a]\right\} .
\end{gathered}
$$

(In order to avoid confusion, in the expression above and in the theorem below we use brackets like $[\cdot, \cdot],\left[\cdot, \cdot\left[\right.\right.$ to denote line segments in a linear space, for example $\left[f^{*}, a^{*}\right]=$ $\left\{\lambda a^{*}+(1-\lambda) f^{*}: 0 \leqslant \lambda \leqslant 1\right\},[a, b[=\{\lambda b+(1-\lambda) a: 0 \leqslant \lambda<1\} ;$ and parenthesis $(\cdot, \cdot)$ are reserved to denote an element of a Cartesian product).

Theorem 4. Let $\varepsilon \in(0,1), \rho \in[1 / 2,1]$. Then, in the space $X=X_{\rho}$ there is $\left(x, x^{*}\right) \in \Pi_{\varepsilon}(X)$, such that for every $\left(y, y^{*}\right) \in S_{X} \times X^{*}$ with $y^{*}(y)=\left\|y^{*}\right\|$ one has $\max \left\{\|x-y\|,\left\|x^{*}-y^{*}\right\|\right\} \geqslant \sqrt{\varepsilon}$.

Proof. We are going to show that $x=(1-\rho \sqrt{\varepsilon}, \rho \sqrt{\varepsilon}), x^{*}=\left(1,1-\frac{\sqrt{\varepsilon}}{\rho}\right)$ is the pair $\left(x, x^{*}\right)$ we are searching for. At first, remark that $x \in] a, b\left[, x^{*} \in\right] a^{*}, f^{*}\left[\right.$ and $x^{*}(x)=1-\rho \sqrt{\varepsilon}+\rho \sqrt{\varepsilon}-$ $\frac{\sqrt{\varepsilon}}{\rho} \cdot \rho \sqrt{\varepsilon}=1-\varepsilon$, so $\left(x, x^{*}\right) \in \Pi_{\varepsilon}(X)$. 
Now assume contrary that there is a $\left(y, y^{*}\right) \in S_{X} \times X^{*}$ with $y^{*}(y)=\left\|y^{*}\right\|$ such that $\max \left\{\|x-y\|,\left\|x^{*}-y^{*}\right\|\right\}<\sqrt{\varepsilon}$. Consider the set $U$ of those $y \in S_{X}$ that $\|x-y\|<\sqrt{\varepsilon}$. $U$ is the intersection of $S_{X}=S_{\rho}$ with the open ball of radius $\sqrt{\varepsilon}$ centered in $x$ ( $U$ is the bold line in Figure 3). The radius of the ball equals to the distance from $x$ to $a$

$$
\|x-a\|=\|(-\rho \sqrt{\varepsilon}, \rho \sqrt{\varepsilon})\|=\rho \sqrt{\varepsilon}+\rho \sqrt{\varepsilon} \cdot \frac{1-\rho}{\rho}=\sqrt{\varepsilon},
$$

which explains the picture for small radius $\sqrt{\varepsilon}$. Also for bigger values of radius the set $U$ can contain points $b$ and $c$, but it never contains any point of $[d, f]$, because the radius $\sqrt{\varepsilon}$ is smaller than 1 , but the distance from $x$ to every point of $[d, f]$ equals 2 . So, $U \subset$ ]$a, b] \cup[b, c] \cup[c, d[$.

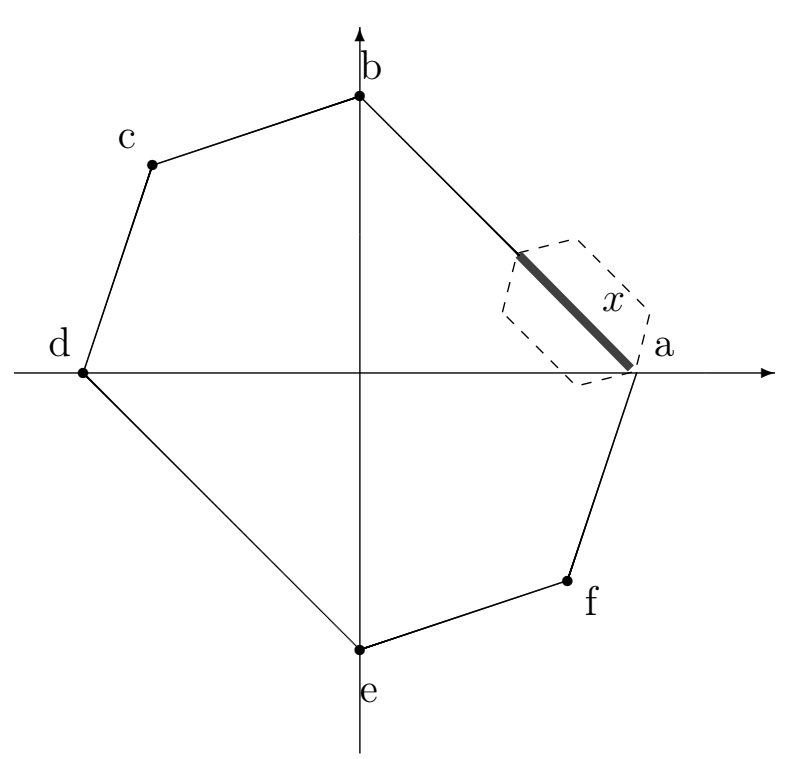

Figure 3

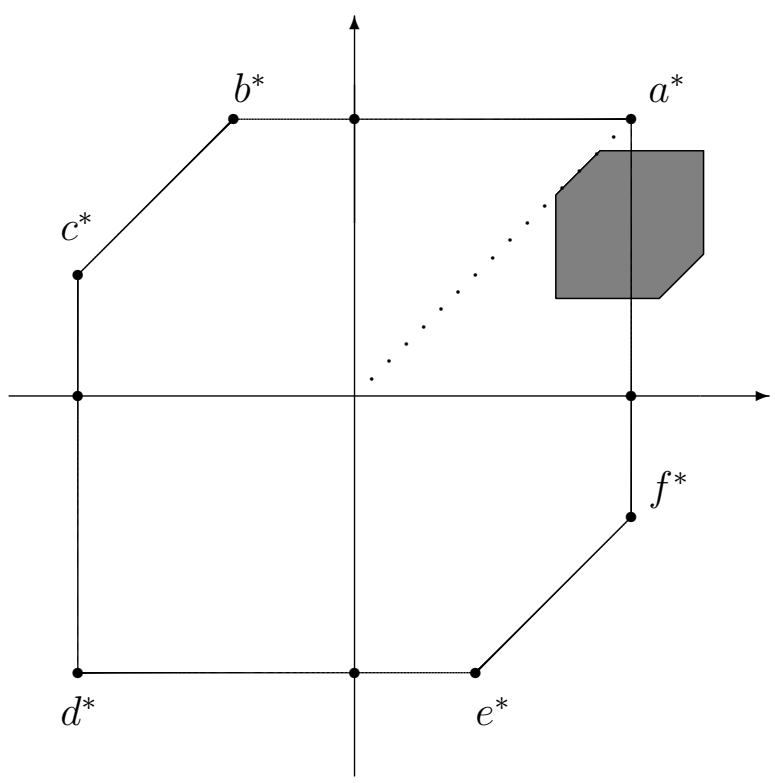

Figure 4

Consider also the set $V$ of those $y^{*} \in X^{*}$ that $\left\|x^{*}-y^{*}\right\|<\sqrt{\varepsilon}$. $V$ is the open ball of radius $\sqrt{\varepsilon}$ centered in $x^{*}$ (the shaded part in Figure 4). In order to explain the picture let us show that the interior of $V$ lies in the open half-plane whose boundary is the principal diagonal (i.e. the set of elements of the form $y^{*}=(\eta, \eta), \eta \in \mathbb{R}$ ). To this end we have to show that $\left\|x^{*}-y^{*}\right\| \geqslant \sqrt{\varepsilon}$ for every $y^{*}=(\eta, \eta)$. In fact,

$$
\left\|x^{*}-y^{*}\right\|=\left\|\left(1-\eta, 1-\eta-\frac{\sqrt{\varepsilon}}{\rho}\right)\right\|=\max \left\{|1-\eta|,\left|1-\eta-\frac{\sqrt{\varepsilon}}{\rho}\right|, \sqrt{\varepsilon}\right\} \geqslant \sqrt{\varepsilon} .
$$

What remains to show is that $\frac{y^{*}(y)}{\left\|y^{*}\right\|} \neq 1$ for every $y \in U$ and every $y^{*} \in V$, or in other words that $\left(y, \frac{y^{*}}{\left\|y^{*}\right\|}\right) \notin \Pi_{0}(X)$. The latter fact follows immediately form the above descriptions of the set $\Pi_{0}\left(X_{\rho}\right)$ and $U$, together with the fact that $\left.\left.\left\{\frac{y^{*}}{\left\|y^{*}\right\|}: y^{*} \in V\right\} \subset\right] d^{*}, e^{*}\right] \cup\left[e^{*}, f^{*}\right] \cup\left[f^{*}, a^{*}[\right.$.

The same way one can prove the sharpness of Lemma 1, and the corresponding example is also not unique. Before stating the result, remark that some values of parameters must be excluded from the sharpness result statement. At first, if $\varepsilon>2$ then $\Pi_{\varepsilon}(X)=\Pi_{2}(X)$, so the rate of approximation in Lemma 1 can be taken as for $\varepsilon=2$, so it can be improved. This 
means that for the sharpness question we must restrict us to $\varepsilon \in(0,2]$. At second, one can easily see that for $k<\frac{\varepsilon}{2}$ the statement is trivial (and is not sharp), because in this case the inequality $\|z-y\| \leqslant \frac{\varepsilon}{k}$ is weaker than the triangle inequality $\|z-y\| \leqslant 2$. Finally, for $k=\frac{\varepsilon}{2}$ the first restriction $\|z-y\| \leqslant \frac{\varepsilon}{k}=2$ is satisfied automatically and $\left\|x^{*}-y^{*}\right\|$ can be chosen arbitrarily small thanks to the Bishop-Phelps theorem.

For the remaining values of parameters Lemma 1 is sharp.

Theorem 5. Let $0<\varepsilon<2, \quad \frac{1}{2} \leqslant \rho<\min \left\{1, \frac{1}{\varepsilon}\right\}$. Then, in the space $X=X_{\rho}$

(i) for every $k \in] \rho \varepsilon, 1\left[\right.$ and every $h<\frac{\varepsilon}{k}$ there is $\left(x, x^{*}\right) \in \Pi_{\varepsilon}(X)$, such that for every $\left(y, y^{*}\right) \in S_{X} \times X^{*}$ with $y^{*}(y)=\left\|y^{*}\right\|$ if $\|x-y\|<h$, then necessarily $\left\|x^{*}-y^{*}\right\|>k$,

(ii) for every $k \in] \rho \varepsilon, 1\left[\right.$ for every $\tilde{h}<k$ there is $\left(x, x^{*}\right) \in \Pi_{\varepsilon}(X)$, such that for every $\left(y, y^{*}\right) \in S_{X} \times X^{*}$ with $y^{*}(y)=\left\|y^{*}\right\|$ if $\left\|x^{*}-y^{*}\right\|<\tilde{h}$, then $\|x-y\|>\frac{\varepsilon}{k}$.

Proof. For (i) one can take $x=(1-\rho h, \rho h), x^{*}=\left(1,1-\frac{\varepsilon}{\rho h}\right)$; for (ii) one can take $x=$ $\left(1-\frac{\rho \varepsilon}{\tilde{h}}, \frac{\rho \varepsilon}{\tilde{h}}\right), x^{*}=\left(1,1-\frac{\tilde{h}}{\rho}\right)$. The pictures and the reasoning are the same as in Theorem 4 .

Acknowledgment. The first author is partially supported by Spanish grants: Junta de Andalucía and FEDER grant FQM-185, MINECO grant MTM2014-57838-C2-1-P and Fundación Séneca, Región de Murcia grant 19368/PI/14. The second author is partially supported by a grant from Akhiezer's Fund, 2015. The authors are thankful to the anonymous referee for his (her) helpful suggestions.

\section{REFERENCES}

1. Bishop E., Phelps R.R., A proof that every Banach space is subreflexive, Bull. Amer. Math. Soc., 67 (1961), 97-98.

2. Bollobás, B., An extension to the theorem of Bishop and Phelps, Bull. London Math. Soc. 2 (1970), 181-182.

3. Cascales B., Kadets V., Guirao A.J., A Bishop-Phelps-Bollobás type theorem for uniform algebras, Advances in Mathematics. 240 (2013), 370-382.

4. Chica M., Kadets V., Martín M., Moreno-Pulido S., Rambla-Barreno F., Bishop-Phelps-Bollobás moduli of a Banach space, J. Math. Anal. Appl., 412 (2014), №2, 697-719.

5. Chica M., Kadets V., Martín M., Merí J., Soloviova, M., Two refinements of the Bishop-Phelps-Bollobás modulus, Banach J. Math. Anal., 9 (2015), №4, 296-315.

6. Diestel J., Geometry of Banach spaces. - Lecture notes in Math., V.485, Springer-Verlag, Berlin, 1975.

7. Phelps R.R., Support cones in Banach spaces and their applications, Adv. Math., 13 (1974), 1-19.

8. Phelps R.R., Convex functions, monotone operators and differentiability (second edition). - Lecture Notes in Math., V.1364, Springer-Verlag, Berlin, 1993.

Department of Mathematics and Informatics

Kharkiv V.N. Karazin National University

v.kateds@karazin.ua; 5_11_16@mail.ru

Received 21.06.2015

Revised 25.09.2015 